exposures at different times are given in the accompanying table.

I wish to express my thanks to Prof. The Svedberg for his interest in this work.

Institute of Physical Chemistry,

Stig Clafesson

University of Uppsala. Nov. 4.

${ }^{1}$ Lamm, O., Nova Acta Reg. Soc. Sci. Upsaliensis, iv, 10, No. 6 (1937).

\section{Computation of Biological Assays}

IN microbiological assays of essential amino-acids, and of members of the vitamin $B_{2}$ complex other than riboflavin and nicotinic acid, it is usually found that when the mean responses are plotted against either the dose or the logarithm of the dose, a nonlinear relationship is obtained. It has been customary to compute the result in such cases by the directreading method, which from the statistical point of view is unsatisfactory in more than one respect, while further examination of the data is difficult or impossible.

On examination of the protocols of several assays by various workers (to whom acknowledgment will be made elsewhere), I find that in most cases the results are fitted well by a straight line, at least over a reasonable range, when the logarithm of the response is plotted against the logarithm of the dose. As an example, reference may be made to a tryptophan assay, the standard curve for which has been published by Barton-Wright ${ }^{1}$. The experimental data plotted in the manner just described are linear over a dosage range of 2-12 $\mu \mathrm{gm}$., or $3 \cdot 75-12 \cdot 6 \mathrm{ml}$. in terms of response.

This 'log-log' transformation provides, for those assays which conform to it, a method of computation which is both sound and simple. The formulæ are precisely the same as in the case, well known in macrobiological assays, in which the response is linearly related to the logarithm of the dose, except that the logarithm of the response is used instead of the response itself. The test and standard lines when plotted on the same graph should theoretically be found to be parallel ; a significant departure from parallelism renders the validity of the assay suspect; and the best estimate of the potency-ratio of the two preparations is obtained from the horizontal distance between the two lines.

Fuller details with illustrative examples will be published later. I should be very interested to receive reports from workers in this field who may test the applicability of the 'log-log' relationship to their own assays. There are further implications which concern the design of assays of this typefor example, doses should be in geometrical rather than arithmetical progression-but this cannot be discussed here.

May I take this opportunity of pointing out that in my previous communication in Nature ${ }^{2}$ about assays in which the response is linearly related to the dose, I neither claimed nor intended to claim that the slope-ratio method of computing them was original. Bliss and Cattell, in a review article ${ }^{3}$ published in 1943, quote three instances of biological assays in which the dose is linearly related to the response. They point out that the slope-ratio method should be used, and add : "such assays are exceptional and their statistical treatment has yet to be described". The best known of the assay techniques they quote is the bradycardia method for aneurin as developed by Harris and his co-workers, the earliest of whose papers is dated $1934^{4}$. More recently, Dr. M. Kerly, in a paper on the riboflavin content of canteen meals ${ }^{5}$, calculated some of her results by the slope-ratio method; her stated reason for doing this is that the line through the test observations did not pass through the 'blank', although the standard line did-an indication that the result may have been statistically invalid. It is clear, however, that the degree of invalidity is not large, and it is a point in favour of the slope-ratio method that the results calculated in this way were found in the three cases in which they were checked by the rat-growth method to be in satisfactory agreement therewith.

Virol Ltd.,

Hanger Lane, Ealing, W.5. Nov. 8.

${ }^{2}$ Barton-Wright, E. C., Analyst, 70, 283 (1945).

Wood, E. C., Nature, 155, 632 (1945).

"Bliss, C. I., and Cattell, McK., "Ann. Rev. Yhysiol.", 5, 479 (1943).

- Birch, T. W., and Harris, L. J., Biochem. J., 28, 602 (1934).

s Kerly, M., Biochem. J., 38, 423 (1944).

\section{Surface Charge of 'Electrets'}

Certarn dielectric materials, if solidified from the molten state in a strong unidirectional electric field, are known to remain in a polarized condition for considerable periods of time and, under certain conditions, surface charges as high as 5 E.S.U. $/ \mathrm{cm} .{ }^{2}$ are retained for several years ${ }^{1-4}$. The materials generally used for the preparation of these so-called 'electrets' are mixtures of carnauba wax and colophony, or carnauba wax, colophony and a small proportion of beeswax.

Several interesting effects have been observed by following the variation with time of the charge on the cathode and anode layers of electrets, these being the surfaces which were adjacent to the negative and positive electrodes, respectively, during preparation. These effects appear to have escaped notice previously, attention having generally been confined to variations in the charge of the cathode layer only.

The preparation consisted in allowing different types of dielectric materials to solidify in a field of approximately $10,000 \mathrm{v} . / \mathrm{cm}$., between two parallel metal electrodes, the tension being maintained for about two hours. When the dielectric had cooled to room temperature, the electrodes were connected to earth for some time to remove temporary surface charges. The samples were then withdrawn, wrapped in tin foil and stored over calcium chloride in a desiccator. Measurements of the surface charges were taken from time to time using a Lindemann electrometer.

Fig. 1 shows typical curves obtained for electrets prepared from two different qualities of prime yellow carnauba wax. It is seen that shortly after the rapid transition from a hetero- to a homo-charge, the anode layer assumes a short-lived high positive charge ; this subsides rapidly to the steady lower charge, which is retained for some time.

In the case of colophony, the charges on the cathode layer were invariably lower and decayed more rapidly than those on the anode layer, when metal electrodes were used in the preparation (Fig. $2, A)$. It is possible that this is due to interaction between the dielectric and the electrodes, or between 